\title{
EXCRECIÓN URINARIA DE ISOFLAVONAS EN ADULTOS MAYORES DE 65 AÑOS QUE CONSUMEN UN ALIMENTO FUNCIONAL
}

\section{URINARY ISOFLAVONE EXCRETION IN ADULTS OLDER THAN 65 YEARS CONSUMING A FUNCTIONAL FOOD}

\author{
Mariane Lutz R. (1,2), Verónica Segura Q. (1), Andrea Cruz V. (3), Andrea López O. (3). \\ (1) Centro de Investigación y Desarrollo de Alimentos Funcionales CIDAF, \\ Facultad de Farmacia, Universidad de Valparaíso \\ (2) Centro Regional de Estudios en Alimentos Saludables - CREAS, Valparaíso, Chile. \\ (3) Tesistas, Carrera de Química y Farmacia, Universidad de Valparaíso.
}

\begin{abstract}
Functional foods (FF) are aimed to exert beneficial health effects. The isoflavones daidzein and genistein are bioactive phytoestrogens that may act as functional food ingredients. This study measured urinary excretion of these isoflavones contained in a powdered novel FF oriented to the elderly population, ready to consume as such or included into culinary preparations. The ingredients containing isoflavones were soybean flour, soybean protein (Glycine max $L$ ) and red clover extract (Trifolium pratense L). The study involved 30 volunteers of both sexes, 60 to 75 years old, who consumed $50 \mathrm{~g}$ of FF (n=16) or placebo food without isoflavones $(n=14)$. The 24-hour urinary excretion of genistein and daidzein were determined before and after the intake of the mentioned foods by HPLC with diode array detector. The 24-h urinary concentration of genistein was $2.06 \pm 1.41$ ppm and the daidzein concentration was $8.80 \pm 4.55 \mathrm{ppm}$. None of the individuals in the placebo group exhibited detectable amounts of isoflavones in urine. The study demonstrates that both phytoestrogens contained in the FF are bioavailable, first step to confirm their ability to exert bioactive effects. The study opens the possibility to verify the effects of both isoflavones to reduce risk factors of non transmissible chronical diseases.
\end{abstract}

Key words: urinary excretion of isoflavones, functional foods, elderly, genistein, daidzein.

Este trabajo fue recibido el 19 de Enero de 2009 y aceptado para ser publicado el 1 de Septiembre de 2009.

\section{INTRODUCCIÓN}

Los adultos mayores (AM), definidos como personas de 65 años de edad o más, constituyen de acuerdo al último censo el 11,4\% de la población chilena (1). Este grupo etáreo es propenso a presentar múltiples problemas nutricionales, ya sea por dificultades económicas, físicas, psicosociales o de otra naturaleza, los que pueden afectar su capacidad para adquirir, preparar y/o consumir una dieta adecuada. Los factores de vulnerabilidad incluyen diversos aspectos cualitativos y cuantitativos de la dieta, cambios fisiológicos, el consumo de alcohol y de medicamentos (polifarmacia), la invalidez, la presencia de patologías asociadas a la vejez, entre otros $(2,3)$. Los únicos alimentos especialmente orientados al AM disponibles actualmente en Chile se distribuyen y comerciali- zan como productos de la línea Años $\operatorname{Dorados}^{R}$, incluida en el Programa de Alimentación Complementaria del Adulto Mayor (PACAM) del Ministerio de Salud (4), y se consumen como una crema o una bebida láctea.

Una estrategia para mejorar la calidad de la alimentación de los AM es la incorporación en su dieta de alimentos funcionales (AF), es decir, aquellos que influyen beneficiosamente sobre una o más funciones del organismo, promoviendo un efecto fisiológico que va más allá del valor nutritivo tradicional, ya sea contribuyendo a la mantención de la salud y bienestar o disminuyendo el riesgo de enfermar (5-7). Frente a la necesidad de contar con este tipo de productos, a través del proyecto FONDEF D01I1069 se desarrolló un AF especialmente orientado a la población mayor 
de 60 años, que incluye a los AM. El producto es de preparación instantánea y puede ser consumido como tal o incorporado en preparaciones de consumo habitual, aportando como ingredientes bioactivos fitoestrógenos estandarizados (8).

Los fitoestrógenos son compuestos polifenólicos que se encuentran en plantas de la subfamilia Papilionoideae en la forma de isoflavonas, dentro de las cuales las más importantes son la genisteína y la daidzeína. Las proporciones relativas de estas especies químicas dependen, en gran medida, del tipo de vegetal y del procesamiento de los productos que las contienen (9). Las principales fuentes alimentarias de isoflavonas son las leguminosas, en particular la soya (Glycine $\max$ L.) y el trébol rojo (Trifolium pratense L.), que fueron las materias primas empleadas en la elaboración del AF evaluado en este trabajo. Las moléculas de genisteína y daidzeína exhiben una similitud estructural y funcional con el $17 \beta$-estradiol, por lo que pueden actuar como agonistas o antagonistas de estrógenos $(10,11)$, y por su estructura química se les ha clasificado como Moduladores Selectivos del Receptor de Estrógeno o SERMs (Selective Estrogen Receptor Modulators) (12). El consumo de isoflavonas se relaciona con efectos saludables, especialmente a nivel de protección cardiovascular, menor incidencia de fracturas óseas asociadas a la osteoporosis y reducción de la incidencia de cáncer de mama, próstata e intestino $(13,14)$.

Un aspecto fundamental en la elaboración de los $\mathrm{AF}$ es asegurar que los agentes bioactivos son biodisponibles, ya que este factor es determinante de su acción saludable. Después de su ingestión, las isoflavonas conjugadas son hidrolizadas a nivel intestinal por $\beta$-glucosidasas, liberando las agliconas daidzeína y genisteína $(10,15)$. Estas son absorbidas directamente, por difusión pasiva, o son transformadas por la microflora intestinal en productos como el equol, dihidrodaidzeína y O-desmetilangolensina (ODMA), derivados de la daidzeína, y p-etilfenol, derivado de la genisteína, los que luego son absorbidos (15-17). Además del metabolismo llevado a cabo por la microflora intestinal, las reacciones de desmetilación pueden ser catalizadas por diferentes citocromos P450. Luego de su absorción, las isoflavonas son transportadas vía porta hacia el hígado, donde son reconjugadas a glucurónidos y sulfatos por enzimas de fase II $(12,16,17)$.

Dada su amplia distribución en los tejidos del organismo, las isoflavonas pueden cuantificarse en plasma, heces, semen, bilis, saliva y leche materna (18). Las especies reconjugadas pueden ser excretadas a través de la bilis y luego reabsorbidas por circulación enterohepática, pero principalmente se excretan inalteradas como conjugados glucurónidos y sulfatos en la orina (19). La daidzeína se elimina en mayor proporción como monoglucurónidos, seguida de sulfoglucurónidos y en forma libre, en tanto que la genisteína se elimina fundamentalmente como monoglucurónidos, seguido de diglucurónidos, sulfoglucurónidos y disulfatos $(20,21)$.

La vida media de eliminación urinaria de la daidzeína y la genisteína es de aproximadamente 4 y 7 h, respectivamente, con máxima excreción entre 6 y 12 h después de la ingesta (21). La biodisponibilidad de estos compuestos se puede determinar a partir de su excreción urinaria de 24 horas, dado que la cantidad acumulativa de isoflavonas en la orina se relaciona directamente con la cantidad absorbida (22).

El metabolismo intestinal de las isoflavonas es afectado por diversos factores, entre los cuales destacan la matriz de los alimentos ingeridos, la magnitud de la fermentación por la microflora bacteriana, el tiempo de tránsito, la presencia de enfermedades intestinales, el uso de antibióticos y la edad, entre otros que pueden afectar la biodisponibilidad y en consecuencia, su potencial bioactivo (23-26).

Se ha señalado que la ingesta mínima de fitoestrógenos necesaria para lograr un efecto beneficioso en los humanos sería de 30 a $50 \mathrm{mg} /$ día, cantidad accesible al ingerir diariamente alimentos que los aportan, tales como la proteína vegetal texturizada de soya y algunos derivados de soya líquidos $(9,27)$. No se cuenta con estimaciones de los valores de la ingesta dietética de fitoestrógenos en la población chilena, en tanto que se ha señalado que en poblaciones europeas es inferior a $1 \mathrm{mg} /$ día, en los norteamericanos se ha estimado que no supera los $5 \mathrm{mg}$ /día y en los asiáticos, grandes consumidores de soya, varía entre 20 y $100 \mathrm{mg} /$ día (28).

El objetivo del presente trabajo es evaluar la excreción urinaria de daidzeína y genisteína, agentes bioactivos contenidos en un nuevo AF en adultos mayores de 60 años, dado que el AF desarrollado está orientado especialmente a este grupo poblacional.

\section{SUJETOS Y MÉTODOS}

El estudio involucró la participación de 30 voluntarios AM de 60 años, chilenos, de ambos sexos, autovalentes, no institucionalizados, que fueron seleccionados mediante una entrevista. Los sujetos fueron divididos aleatoriamente en dos grupos, uno de los cuales consumió el AF en estudio $(n=14)$ y el otro actuó como grupo placebo $(n=16)$, que consumió un alimento de idéntica composición y presentación, pero carente de los ingredientes aportadores de isoflavonas.

La selección de los voluntarios participantes exigió que presentaran un índice de masa corporal (IMC) 
normal y valores de creatinuria normal. Se excluyeron los sujetos que padecieran de enfermedades renales, hepáticas o gastrointestinales severas diagnosticadas, los que estuvieran en tratamiento con antibióticos (hasta dos meses antes de la prueba) o con diuréticos, ya que éstos pueden interferir en la biodisponibilidad de las isoflavonas. Asimismo, se excluyeron los sujetos que en los dos días previos al estudio hubieran consumido alimentos que sean fuente reconocida de isoflavonas, para evitar una sobreestimación de los resultados. Todos los voluntarios presentaron características fisiológicas compatibles con la adecuada determinación de la excreción urinaria de las isoflavonas. El diseño experimental fue aprobado por el Comité de Etica de la Facultad de Farmacia de la Universidad de Valparaíso. A cada sujeto se le solicitó su participación mediante un consentimiento informado que incluyó para ambos grupos (AF y placebo) la aclaración que la participación en el estudio no comprometía la obtención de un beneficio saludable en particular.

El AF empleado en el estudio fue elaborado a base de una mezcla de harinas de cereales y leguminosas y una premezcla de vitaminas y minerales. Los ingredientes aportadores de isoflavonas son harina de soya entera preextruída y proteína de soya comercial. Todas las demás harinas se incorporaron en forma preextruída, apta para una preparación instantánea. También se adicionó un extracto estandarizado de trébol rojo, en el cual la daidzeína y la genisteína se encuentran como una mezcla de agliconas, glucósidos, acetilglucósidos y malonilglucósidos, predominando los $\beta$-glicósidos conjugados, que no son biodisponibles, en las formas de daidzina y genistina, respectivamente (9). El AF se presenta como un producto en polvo de reconstitución instantánea al $25 \%$, en que una porción se reconstituye en agua para formar una sopa o bien se incorpora en diversos guisos o preparaciones culinarias (8). Este AF fue producido a nivel de planta piloto en la planta de la empresa IPAK, Quilpué.

Los voluntarios ingirieron una porción única de 50 $\mathrm{g}$ de alimento (AF o placebo), preparado en forma de una sopa crema instantánea en $200 \mathrm{~mL}$ de agua recién hervida, durante la hora de almuerzo. Se tomó una muestra de orina basal previo al consumo del alimento y posteriormente se recolectó la orina total durante las $24 \mathrm{~h}$ siguientes. Las personas fueron capacitadas para este proceso y se les entregó los materiales requeridos para la recolección. Se midió el volumen total de orina, reservando 3 alícuotas de la orina de cada sujeto almacenadas a $-20^{\circ} \mathrm{C}$ hasta su análisis.

Para la cuantificación de genisteína y daidzeína en el AF y en la orina recolectada se empleó el método de Franke $(29,30)$ optimizado (31). En la extracción de las isoflavonas a partir de las muestras se empleó $\beta$-glucuronidasa ( $\beta$-D-glucurónido glucuronosohidrolasa, EC 3.2.1.31) aislada de Helix pomatia tipo HP-2S (Sigma). En la identificación y cuantificación se utilizó un cromatógrafo líquido de alta resolución (HPLC) LaChrom ${ }^{\circledR}$ Merck-Hitachi equipado con detector de arreglo de diodo. Se emplearon columnas de extracción en fase sólida C18. Como estándares se empleó daidzeína (4',7-dihidroxisoflavona; $\mathrm{C} 15 \mathrm{H} 10 \mathrm{O} 4)$ de $98 \%$ de pureza (Sigma) y genisteína (4', 5, 7-trihidroxiisoflavona; C15H10O5) de $98 \%$ de pureza (Sigma). Como patrón interno se empleó flavona (2-fenilcromona; C15H10O2) (Sigma). Las condiciones cromatográficas empleadas fueron: $260 \mathrm{~nm}$, volumen de inyección $20 \mu \mathrm{L}$, flujo: 1,5 $\mathrm{mL} / \mathrm{min}$, con fase móvil de ácido acético / acetonitrilo.

Los resultados se presentan como promedio + desviación estándar. Dado que las isoflavonas sólo fueron detectadas en la orina de los voluntarios participantes en el estudio que ingirieron AF, no se aplica estadística comparativa entre los grupos experimentales (casos versus placebo).

\section{RESULTADOS}

Los 14 voluntarios que ingirieron AF (10 mujeres, 4 hombres) presentaron una edad promedio de 67años, con un rango de 60 a 75 años. Su IMC se encontró en el rango de 23,62 a $31,14 \mathrm{~kg} / \mathrm{m}^{2}$ y los valores de creatinuria eran normales, con un rango de 597 a $750 \mathrm{mg} / 24 \mathrm{~h}$ en mujeres y 967 a $1613 \mathrm{mg} / 24 \mathrm{~h}$ en hombres.

Previo al análisis de los niveles de isoflavonas en la orina de los voluntarios que consumieron el nuevo AF, se midió el contenido de genisteína y daidzeína en el alimento, para conocer la cantidad contenida en la porción consumida, de $50 \mathrm{~g}$, que corresponde a lo que se recomienda ingerir al día. Las cantidades de isoflavonas contenidas en el AF fueron de 0,35 $\pm 0,02 \mathrm{mg} /$ $\mathrm{kg}$ de daidzeína y $0,48 \pm 0,02 \mathrm{mg} / \mathrm{kg}$ de genisteína. En el alimento placebo no se detectó la presencia de estas isoflavonas.

La concentración urinaria promedio de daidzeína y genisteína luego del consumo del AF fue de 8,81 \pm 4,55 ppm y de 2,06 $\pm 1,41 \mathrm{ppm}$, respectivamente. Pese a las diferencias interindividuales observadas, en todos los sujetos que consumieron el AF se cuantificaron las isoflavonas de interés. Al analizar los valores obtenidos por sexo, se observó que las concentraciones de daidzeína y genisteína en los hombres $(\mathrm{n}=4)$ alcanzaron a $12,12 \pm$ $5,85 \mathrm{ppm}$ y $3,24 \pm 1,78 \mathrm{ppm}$, respectivamente, en tanto que en las mujeres $(n=10)$ fueron $7,48 \pm 3,65 \mathrm{ppm}$ y 1,58 $\pm 0,85 \mathrm{ppm}$, respectivamente (Figura 1).

Las concentraciones de daidzeína y genisteína en 
las muestras de orina basal, es decir, aquella que se obtuvo antes de la ingesta del alimento, tanto en los voluntarios que consumieron el AF como el placebo, fueron inferiores al límite de cuantificación para ambas isoflavonas. Ninguno de los voluntarios había ingerido alimentos posibles fuentes de isoflavonas los días previos al estudio, lo cual es corroborado por este resultado. Es interesante no haber detectado la presencia de isoflavonas en las muestras de orina de $24 \mathrm{~h}$ de los consumidores de placebo, a pesar de que este alimento contenía la misma base que el AF, una mezcla de harinas de cereales y leguminosas, por lo que podría haberse esperado medir niveles bajos de isoflavonas. Ello posiblemente no ocurrió debido a que el contenido de estos compuestos en las leguminosas contenidas en la porción ingerida (50 g) se encuentra por debajo del límite de detección instrumental.

\section{DISCUSIÓN}

En el diseño y la elaboración de un AF se debe considerar que los compuestos bioactivos contenidos en el producto deben ser biodisponibles para que logren afectar beneficiosamente la salud de quien los consume. En este estudio se formuló un AF que contiene fitoestrógenos, especialmente orientado al AM. En consecuencia, el primer paso para verificar si el AF cumple con su objetivo es determinar si los compuestos bioactivos son absorbidos y luego excretados del organismo es decir, son biodisponibles. Una forma de evidenciar este proceso es a través de la medición de las isoflavonas de interés en la orina excretada en un período de tiempo que asegure que se cumplieron a cabalidad los procesos de digestión, metabolismo intestinal, absorción, distribución y finalmente excreción por la vía urinaria. Por esta razón, se determinó la excreción urinaria de 24 horas de daidzeína y genisteína, los dos compuestos bioactivos contenidos en el AF, en los voluntarios que consumieron el AF de prueba (32).

Las concentraciones de isoflavonas observadas en la orina de los diferentes sujetos presentaron una gran dispersión, lo que es concordante con los datos bibliográficos. Esta alta intervariabilidad se atribuye a que en el metabolismo de los fitoestrógenos la microflora intestinal tiene un papel crucial, la que depende de múltiples factores (12,14-16), sumado a efectos del tiempo de tránsito intestinal, $\mathrm{pH}$ y estado redox del intestino (21), todo lo cual se encuentra a su vez bajo la influencia de la dieta, posible uso previo de fármacos, situación inmunitaria, así como la matriz alimentaria que contiene las isoflavonas, entre otros $(24-26,33)$.

\section{FIGURA 1}

\section{Concentración de isoflavonas en orina de $\mathbf{2 4} \mathrm{h}$ de adultos mayores voluntarios que consumieron alimento funcional, según sexo $(n=14)$}

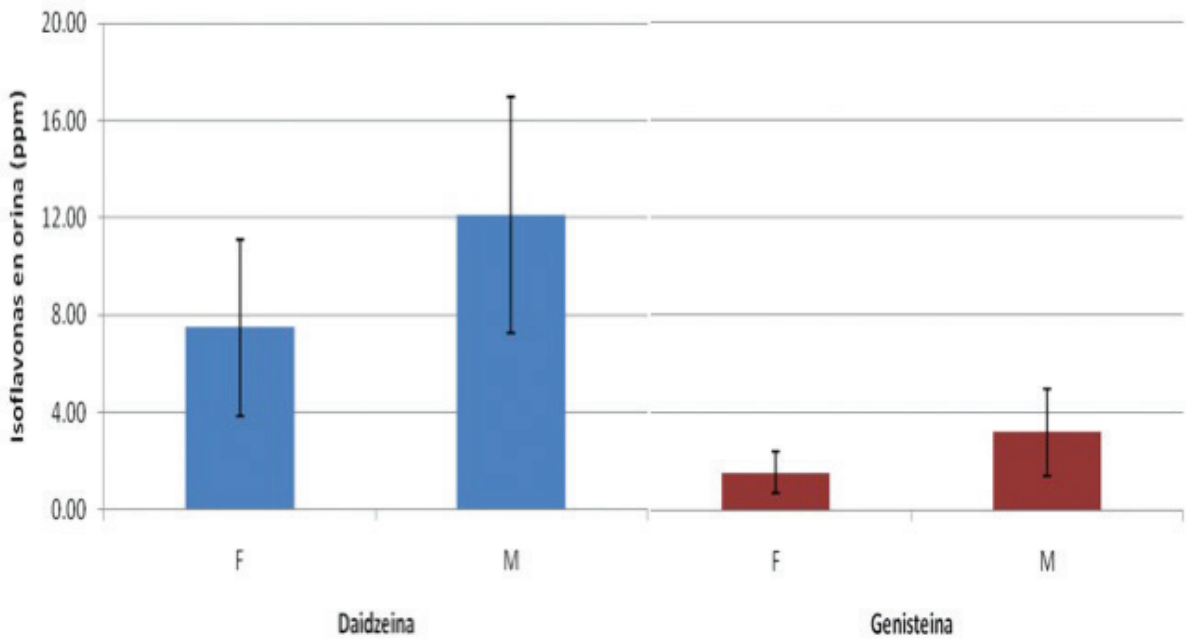

Las barras indican los valores promedio y las líneas muestran la DE

(F): Femenino, (M): Masculino 
En este estudio se incluyeron sujetos de ambos sexos debido a que el AF está orientado a producir efectos saludables en toda la población, en términos de reducción de factores de riesgo de enfermedades crónicas no transmisibles, por lo que es de interés que las isoflavonas sean biodisponibles en todos los consumidores. La alta variabilidad interindividual observada entre los voluntarios es responsable de que los niveles excretados de las isoflavonas en la orina de $24 \mathrm{~h}$ no acusara diferencias según sexo. No obstante lo anterior, este análisis de los resultados muestra, en primer término, que en todos los participantes en el estudio los niveles de daidzeína son más altos que los de genisteína, lo que concuerda con el mayor contenido de esta isoflavona en el AF consumido y, en segundo término, que los hombres tienden a excretar mayor cantidad de isoflavonas por la vía urinaria, lo cual es consistente con datos de la literatura $(19,21,22,32)$.

Estos resultados ponen en evidencia que las isoflavonas contenidas en el nuevo AF son biodisponibles, lo cual es determinante para que estos compuestos bioactivos ejerzan su esperado efecto saludable. En consecuencia, este AF representa una buena alternativa para lograr que el consumo dietético de fitoestrógenos alcance niveles compatibles con un efecto beneficioso en la salud del consumidor. Queda de manifiesto que la matriz del AF, constituida fundamentalmente por una mezcla de harinas de cereales y leguminosas, adicionada de mezcla de vitaminas y minerales, no interfiere significativamente con la capacidad de absorber y utilizar las isoflavonas bioactivas de interés. Es así que la recuperación urinaria de las isoflavonas se encuentra en rangos compatibles con la finalidad de un AF, esto es, contribuir al aporte de agentes bioactivos, sin constituir per se la única fuente de ellos, promoviendo el consumo de una alimentación variada de carácter saludable.

\section{RESUMEN}

Los alimentos funcionales (AF) ejercen efectos beneficiosos en la salud. Las isoflavonas daidzeína y genisteína son fitoestrógenos bioactivos que pueden actuar como ingredientes alimentarios funcionales. En este trabajo se evalúa la excreción urinaria de 24 horas de estas isoflavonas contenidas en un nuevo $\mathrm{AF}$ en polvo, de preparación instantánea, para ser consumido solo o incorporado en preparaciones, especialmente orientado a adultos mayores. Los ingredientes aportadores de isoflavonas son harina de soya, proteína de soya (Glycine max L.) y extracto de trébol rojo (Trifolium pratense $L$.). El estudio se realizó en 30 voluntarios seleccionados de ambos sexos, de 60 a 75 años, divididos aleatoriamente en dos grupos, que consumieron una porción de $50 \mathrm{~g}$ de alimento funcional $(\mathrm{n}=16)$ o alimento placebo sin ingredientes aportadores de isoflavonas $(n=14)$. La excreción urinaria de daidzeína y genisteína se determinó en la orina de 24 horas antes de la ingesta y posterior a la ingestión del alimento, y fueron cuantificadas por HPLC con detector de arreglo de diodo. La concentración urinaria de genisteína fue 2,06 $\pm 1,41 \mathrm{ppm}$ y la de daidzeína fue 8,80 $\pm 4,55 \mathrm{ppm}$. En el grupo placebo las isoflavonas no fueron detectadas en la orina. El estudio muestra que las isoflavonas ingeridas a través del AF son biodisponibles, primer paso para poner en evidencia su carácter bioactivo. El trabajo abre la posibilidad de verificar los efectos de las isoflavonas contenidas en estos AF sobre la disminución del riesgo de enfermedades crónicas no transmisibles.

Palabras clave: Excreción urinaria de isoflavonas; alimentos funcionales; adulto mayor; genisteína, daidzeína.

Dirigir la correspondencia a:

Profesora

Mariane Lutz R.

Centro de Investigación y Desarrollo de

Alimentos Funcionales CIDAF

Facultad de Farmacia,

Universidad de Valparaíso.

Av. Gran Bretaña 1093

Valparaíso. Chile

Teléfono: 56-032-2508418.

Fax 56-032-2508111

E-mail: mariane.lutz@uv.cl

Agradecimientos: Financiado por proyectos FONDEF D01I1069 y CID 04/06.

\section{BIBLIOGRAFÍA}

1. Instituto Nacional de Estadísticas. XVII Censo Nacional de Población 2002. Disponible en: www. ine.cl/canales/chile_estadistico/estadisticas_sociales_culturales/adultosmayores/adultos_mayores. php. Consultado 20 agosto 2009.

2. Ausman L, Russell R. Nutrición en el anciano. En: Nutrición en salud y enfermedad. Shils M, Olson J, Shike M y Ross, C. $9^{a}$ Edición. Editorial Mc Graw Hill Interamericana, México, 2002, pp 997-1011.

3. Sabartés O. Factores de riesgo de malnutrición. En: Rubio Herrera MA, editor. Manual de alimentación y nutrición en el anciano. Masson, Barcelona, 2002, p. 31-38.

4. Ministerio de Salud, República de Chile. Programa de Alimentación Complementaria del Adulto Mayor (PACAM). Disponible en: www.minsal.cl. Consul- 
tado 20 agosto 2009.

5. Lutz M. Alimentos funcionales en la prevención de enfermedades crónicas no transmisibles. En: Lutz M, León AE, editores. Aspectos nutricionales y saludables de los productos de panificación, Universidad de Valparaíso Editorial, 2009, pp. 39-51.

6. Araya H, Lutz M. Alimentos funcionales y saludables. Rev Chil Nutr. 2003; 30: 8-14.

7. Halsted C. Dietary supplements and functional foods: 2 sides of a coin? Am J Clin Nutr 2003; 77(suppl): 1001S-1007S.

8. Lutz M, Morales D, Sepúlveda S, Alviña M. Evaluación sensorial de preparaciones elaboradas con nuevos alimentos funcionales destinados al adulto mayor. Rev Chil Nutr 2008; 35: 131-137.

9. Zubik L, Meydani M. Bioavailability of soybean isoflavones from aglycone and glucoside forms in American women. Am J Clin Nutr 2003; 77: 14591465.

10. Dixon RA. Phytoestrogens. Annu Rev Plant Biol 2004; 55: 225-261.

11. Song WO, Chun OK, Hwang I, Shin HS, Kim BG, Kim KS, Lee SY, Shin D, Lee SG. Soy isoflavones as safe functional ingredients. J Med Food 2007; 10: 571-580.

12. Setchell K. Soy isoflavones. Benefits and risks from nature's selective estrogen receptor modulators (SERMs). J Am Coll Nutr 2001; 20: 354S-362S.

13. Setchell KD, Brown NM, Zimmer-Nechemias L, Brashear WT, Wolfe BE, Kirschner AS, Heubi JE. Evidence for lack of absorption of soy isoflavone glycosides in humans, supporting the crucial role of intestinal metabolism for bioavailability. Am J Clin Nutr 2002; 76: 447-453.

14. Cassidy A, Albertazzi P, Lise Nielsen I, Hall W, Williamson G, Tetens I, Atkins S, Cross H, Manios Y, Wolk A, Steiner C, Branca F. Critical review of health effects of soybean phytoestrogens in postmenopausal women. Proc Nutr Soc 2006;65:76-92.

15. Atkinson C, Frankenfeld CL, Lampe JW. Gut bacterial metabolism of the soy isoflavone daidzein: exploring the relevance to human health. Exp Biol Med 2005; 230: 155-170.

16. Hu M, Krausz K, Chen J, Ge X, Li J, Gelboin H, Gonzalez F. Identification of CYP1A2 as the main isoform for the phase I hydroxylated metabolism of genistein and a prodrug converting enzyme of methylated isoflavones. Drug Metab Dispos 2003; 31: 924-931.

17. Richelle M, Pridmore-Merten S, Bodenstab S, Enslen M, Offord EA. Hydrolysis of isoflavone glycosides to aglycones by $\square$-glycosidase does not alter plasma and urine isoflavone pharmacokinetics in postmenopausal women. J Nutr 2002; 132: 25872592.

18. Kano M, Takayanagi T, Harada K, Sawada S, Ishikawa F. Bioavailability of isoflavones after ingestion of soy beverages in healthy adults. J Nutr 2006; 136: 2291-2296.

19. Prasain JK, Barnes S. Metabolism and bioavailability of flavonoids in chemoprevention: Current analytical strategies and future prospectus. Mol Pharm 2007; 4: 846-863.

20. Zhang Y, Hendrich S, Murphy P. Glucuronides are the main isoflavone metabolites in women. J Nutr 2003; 133: 399-404.

21. Setchell KRD, Brown NM, Desai P, ZimmerNechemias L, Wolfe BE, Jakate AS, Creutzingerb V, Heubi JE. Bioavailability, disposition, and doseresponse effects of soy isoflavones when consumed by healthy women at physiologically typical dietary intakes. J Nutr 2003; 133: 1027-1035.

22. Ritchie MR, Morton MS, Thompson AM, Deighton $\mathrm{N}$, Blake A, Cummings JH, Steel CM. Investigation on the reliability of $24 \mathrm{~h}$ urine excretion as a biomarker of isoflavone intakes. Eur J Clin Nutr 2004; 58: 1286-1289.

23. Munro IC, Harwood M, Hlywka H, Stephen AM, Doull J, Flamm WG, Adlercreutz H. Soy Isoflavones: A Safety Review. Nutr Rev 2003; 61: 1-33.

24. Uzzan M, Labuza TP. Critical issues in R\&D of soy isoflavone-enriched foods and dietary supplements. J Food Sci 2004; 69: 77-86.

25. Cassidy A, Brown JE, Hawdon A, Faughnan MS, King LJ, Millward J, Zimmer-Nechemias L, Wolfe B, Setchell KDR. Factors affecting the bioavailability of soy isoflavones in humans after ingestion of physiologically relevant levels from different soy foods. J Nutr 2006; 136: 45-51.

26. Setchell K. Absorption and metabolism of soy isoflavones from food to dietary supplements and adults to infants. J Nutr 2000; 130: 654S-655S.

27. Hendrich S. Bioavailability of isoflavones. J Chromatogr B 2002; 777: 203-210.

28. van Erp-Baart MAJ, Brants HAM, Kiely M, Mulligan A, Turrini A, Sermoneta C. Kilkkinen A, Valsta LM. Isoflavone intake in four different European countries: the VENUS approach. Brit J Nutr 2003; 89 Suppl 1; 815-821.

29. Franke A, Custer L, Wang W, Shi C. HPLC analysis of isoflavonoids and other phenolic agents from foods and from human fluids. Proc Soc Exp Biol Med 1998; 217: 263-273.

30. Franke A, Custer L. High-performance liquid chro- 
matographic assay of isoflavonoids and coumestrol from human urine. J Chromatogr B 1994; 662: 47-60.

31. Márquez P. Optimización y validación de un método HPLC para la determinación de fitoestrógenos en alimentos. Internado para optar al título de Químico Farmacéutico de la Universidad de Valparaíso, 2005.
32. Spencer JPE, Abd El Mohsen MM, Minihane AM, Mathers JC. Biomarkers of the intake of dietary polyphenols: strengths, limitations and application in nutrition research. Brit J Nutr 2008; 99: 12-22.

33. de Pascual-Teresa S, Hallund J, Talbot D, Schroot J, Williams CM, Bugel S, Cassidy A. Absorption of isoflavones in humans: effects of food matrix and processing. J Nutr Biochem 2006; 257-264. 